\title{
Outcomes of Combined Prolene Gonioscopy Assisted Transluminal Trabeculotomy with Phacoemulsification in Open-Angle Glaucoma
}

This article was published in the following Dove Press journal:

Clinical Ophthalmology

Waldo Loayza-Gamboa $\mathbb{D}^{1,2}$ Vladimir Martel-Ramirez $\mathbb{D D}^{1,3}$ Vanessa Inga-Condezo (D) ${ }^{1,3}$ Vanessa Valderrama-Albino ${ }^{2,3}$ Rosa Alvarado-Villacorta ${ }^{2,3}$ Diego Valera-Cornejo (iD ${ }^{2,3}$

'Glaucoma Department, Instituto Nacional de Oftalmología "Francisco Contreras Campos", Lima, Perú; ${ }^{2}$ Research unit, Clínica Vista, Lima, Perú; ${ }^{3}$ Faculty of medicine, Universidad Peruana Cayetano Heredia, Lima, Peru
Correspondence: Diego Valera-Cornejo Vista Clinic, Jirón Caracas 2410 Jesús

María, Lima, Perú

Tel +5I I 64I32I7

Email diegoavalera@gmail.com
Purpose: To describe the clinical outcomes of combined prolene gonioscopy assisted transluminal trabeculotomy (GATT) with phacoemulsification in patients with open-angle glaucoma.

Methods: Retrospective study of patients with cataract and open-angle glaucoma treated with GATT and phacoemulsification with intraocular lens (IOL) implantation. Patients with neovascular glaucoma, corneal decompensation, unstable IOL, and with bleeding disorders were excluded. The cumulative probability of failure was the primary outcome. Secondary outcomes were the complete success rate, the mean change on intraocular pressure (IOP), best-corrected visual acuity (BCVA) and number of glaucoma medications at the last followup. The frequency of complications was also recorded.

Results: Thirty two eyes (25 patients) were included. Nineteen (59.3\%) were females, with a mean age of $73.4 \pm 12.4$ (23-87 years) and a mean baseline BCVA of $1.1 \pm 0.75(0.3-3)$ LogMAR units. The cumulative probability of failure was $3.2 \%$ at 1 month and $6.3 \%$ at 6 and 12 months. Sixty-eight and $65.5 \%$ of eyes achieved complete success at 3 and 6 months, respectively. The reduction of IOP and number of glaucoma medications at 6 months were statistically significant $(\mathrm{p}<0.0001$ and $\mathrm{p}=0.0002$, respectively). There was a statistically significant improvement of BCVA (from $1.1 \pm 0.7$ to $0.4 \pm 0.7$ ) at 6 months $(\mathrm{p}<0.0001$ ). IOP spikes $(18.7 \%)$ and transient hyphema $(9.3 \%)$ were the most common complications.

Conclusion: Combined GATT with cataract surgery reduced the IOP and was associated with low failure rates and few ocular complications. This procedure offers the advantage to avoid the need for a bleb, scleral, or conjunctival incision, allowing the possibility for future glaucoma procedures.

Keywords: glaucoma surgery, cataract surgery, Gonioscopy-assisted, trabeculotomy, ab interno, transluminal

\section{Introduction}

Glaucoma leads to irreversible damage to the ganglion cells and is the second cause of irreversible blindness in the world. ${ }^{1}$ In order to reduce the risk of visual loss due to glaucoma, it is necessary to decrease the intraocular pressure (IOP) by using topical antiglaucomatous medications, laser, or surgery. ${ }^{2-5}$ Trabeculotomy was $^{2}$ described in 1969 as an external procedure for treating several types of glaucoma (congenital, juvenile, open-angle, pseudoexfoliation). ${ }^{6-9}$ This procedure lowers IOP by improving the aqueous flow through the Schlemm's canal and collector channels without the formation of a conjunctival bleb. ${ }^{10}$ Over the last years, this procedure 
has switched to a minimally invasive ab interno procedure. Grover et al first described Gonioscopy-assisted transluminal trabeculotomy (GATT) in 2014 for the treatment of open-angle glaucoma. ${ }^{11,12}$ This blebless and sutureless procedure fractures the trabecular meshwork, decreasing the proximal resistance of the conventional outflow system and it has been shown to significantly reduce the IOP in open-angle glaucoma as well as juvenile and congenital glaucoma. $^{11,12} \mathrm{~A}$ major advantage that offers a nonpenetrating procedure is the potential for fewer complications. Since there is no fistula through the sclera, complications such as hypotony with a flat anterior chamber and choroidal detachments are avoided. Additionally, blebs and its associated problems (leakage, discomfort, and infections), commonly seen after trabeculectomy, are also avoided. In addition, we believe that an advantage of this technique is that it can be performed safely with a 5-0 prolene suture, which costs approximately 5 USD. This means that this surgery can be performed effectively with very low costs, which would have big economic implications for glaucoma care in developing countries. Herein, we aimed to describe the IOP lowering effect and safety of GATT combined with phacoemulsification and intraocular lens implantation in patients with moderate to advanced open-angle glaucoma.

\section{Materials and Methods \\ Study Design}

In this retrospective study, medical records from patients with diagnosis of cataract and open-angle glaucoma treated with GATT and phacoemulsification with intraocular lens (IOL) implantation at the glaucoma service of the National Eye Institute of Peru during January 1, 2018 to December 2019, were included. Institutional Review Board approval from the National Eye Institute of Peru (RCEI-56) ethics committee was obtained, and the study followed the tenets of the Declaration of Helsinki. Informed patients' consent was waived because of the retrospective design and the accessed patient data complied with relevant data protection and privacy regulations.

\section{Study Participants}

A total of 32 eyes from 25 patients were enrolled. The diagnosis in all cases was made clinically, and preoperative gonioscopy was performed to verify the visibility of trabecular meshwork. Patients older than 18 years, with medically uncontrolled open-angle glaucoma (IOP levels above target or evidence of glaucoma progression due to visual field progression or retinal nerve fiber layer loss) and any significant cataract with best-corrected visual acuity (BCVA) worse than 20/40 were included. Patients with self-reported difficulties in daily activities (driving and reading), due to visual impairment caused by cataract, who were on maximum medical therapy for IOP control, were also included. Patients with angle closure glaucoma, neovascular glaucoma, corneal endothelial decompensation, unstable IOL, those in whom angular structures (trabecular meshwork) cannot be identified and patients with bleeding disorders or those who were unable to stop anticoagulant medications were excluded. Medical records were reviewed for documentation of baseline demographic and clinical details. Follow-up evaluations were performed on days 1, 7 and at 1, 3, 6- and 12-months post-treatment. Best-corrected visual acuity (BCVA; reported in LogMAR units for statistical purposes), IOP levels [measured by the Goldmann applanation tonometry (AT900, Haag-Streit, Köniz, Switzerland)], anterior segment assessment, and the number of glaucoma medications were recorded at each visit.

\section{Surgical Procedure}

All surgeries were performed by one surgeon (W.L.G.) and phacoemulsification with IOL implantation was performed before GATT. A $2.4 \mathrm{~mm}$ clear corneal incision was made in the superior (12 o'clock meridian) quadrant; then, an ophthalmic viscosurgical device (OVD) was injected into the anterior chamber and two additional corneal paracentesis were made (upper nasal and temporal quadrants). Capsulorhexis was created and phacoemulsification was performed with a foldable IOL that was implanted in the bag. Balanced salt solution (BSS) was then injected to fill the anterior chamber. An additional paracentesis was made in the lower temporal quadrant to place an anterior chamber maintainer connected to an infusion bottle (Balanced Salt solution) at a height of $90 \mathrm{~cm}$ from the patient's eye. The patient's head and microscope were tilted to provide visualization of the nasal iridocorneal angle. Using the surgical goniolens (AVG; Surgical Gonio Lens, Volk Alcon, Mentor, $\mathrm{OH}, \mathrm{USA}$ ) a $1-2 \mathrm{~mm}$ goniotomy was made on the nasal angle using a $23-\mathrm{G}$ needle trough the temporal paracentesis. A 6-0 prolene suture was blunted using a cautery and was directed toward the nasal angle via the superior nasal paracentesis. Microsurgical forceps (Grieshaber $^{\circledR}$ Maxgrip ${ }^{\text {TM }} 23 \mathrm{G}$ forceps, Alcon, Fort Worth, TX) was then introduced through the temporal 
paracentesis and used to grasp the blunted suture within the anterior chamber, the tip of the suture is then inserted into the Schlemm canal at the nasal goniotomy incision. The microsurgical forceps are used to advance the suture through the canal circumferentially 360 degrees. The trabeculotomy was performed by pulling both ends of the suture. If it was not possible to canalize the 360 degrees of the canal, a trabeculotomy (of at least 100 degrees) was performed up to the area of the Schlemm's canal stenosis and maneuvers were attempted to achieve as close to 360 degrees as possible. OVD was then removed from the anterior chamber, the infusion bottle was raised $10 \mathrm{~cm}$ and balanced salt solution was injected inflating the anterior chamber to achieve an episcleral venous fluid wave (EVFW) with blanching of the limbal area. No OVD was left in the anterior chamber at the end of the surgery, the main wound was hydrated to ensure a watertight seal and the anterior chamber was filled with BSS to increase the IOP. Post-operative medications included topical moxifloxacin four times daily for 1 week and topical prednisolone acetate five times daily, which was then tapered over a period of 8 weeks.

\section{Outcome Measures}

The primary outcome was the cumulative probability of failure to the treatment which was defined as any of the following: IOP $\geq 18 \mathrm{mmHg}$ or $<5 \mathrm{mmHg}$, or $<20 \%$ IOP reduction from baseline value for two consecutive visits, the needed of an additional glaucoma surgery, or the complete loss of light perception. Complete success was defined as the absence of failure to treatment without the use of glaucoma medications, while qualified success allowed the use of glaucoma medications. Secondary outcomes were the frequency of complete success, the mean change on IOP, BCVA and number of glaucoma medications at the last follow-up. The frequency of complications such as hyphema (defined as a visible layered blood cloth), IOP spikes, choroidal detachment and corneal edema were also recorded.

\section{Statistical Analysis}

Statistical analysis was performed using Stata $\subset$ software version 15.1 (StataCorp. 2015, Stata Statistical Software: Release 15. College Station, Texas, US: StataCorp LP) and GraphPad Prism software Version 8.4.2 (GraphPad Software Inc, La Jolla, CA). The normal distribution of variables was assessed with Shapiro-Wilk test and $p<0.05$ was considered significant. Continuous variables were expressed as means \pm standard deviation (SD) or median and interquartile range (IQR). Categorical variables were expressed as absolute (n) and relative frequencies (\%). Time-to-event curve was generated using the KaplanMeier method to estimate the probability of failure. Comparisons were also performed, using the Wilcoxon test. A p-value $<0.05$ was considered statistically significant.

\section{Results}

We included 32 eyes from 25 patients, 19 (59.3\%) eyes were from female patients, with a mean age of $73.4 \pm 12.4$ (23-87 years) and a mean baseline BCVA of $1.1 \pm 0.75$ (0.3-3) LogMAR. Primary open-angle glaucoma was the most frequent diagnosis (84.3\%) followed by pseudoexfoliation glaucoma (9.5\%). Table 1 summarizes baseline characteristics of baseline BCVA, IOP level, and other variables of interest.

The cumulative probability of failure was $3.2 \%$ at 1 month and $6.3 \%$ at 6 and 12 months (Figure 1). Sixty-eight and $65.5 \%$ of eyes achieved complete success at 3 and 6 months, respectively. The reduction of IOP levels and number of glaucoma medications at 6 months were statistically significant $(\mathrm{p}<0.0001$ and $\mathrm{p}=0.0002$, respectively) (Figures 2 and 3). The mean change in IOP was $-13.3 \pm$

Table I Baseline Demographic Characteristics $(n=32)$

\begin{tabular}{|c|c|}
\hline Variable & Value \\
\hline Age (years), $\bar{x} \pm$ SD (Range) & $73.4 \pm 12.4(23-87)$ \\
\hline \multicolumn{2}{|l|}{ Sex, n (\%) } \\
\hline Female & $19(59.3)$ \\
\hline \multicolumn{2}{|l|}{ Diagnosis } \\
\hline Primary open-angle glaucoma & $27(84.3)$ \\
\hline Pseudoexfoliation glaucoma & $3(9.5)$ \\
\hline Uveitic/inflammatory glaucoma & I (3.1) \\
\hline Juvenile glaucoma & $\mathrm{I}(3.1)$ \\
\hline $\begin{array}{l}\text { Baseline cup/disc ratio, } \bar{x} \pm S D \\
\text { (range) }\end{array}$ & $0.82 \pm 0.10(0.8-0.99)$ \\
\hline $\begin{array}{l}\text { Baseline glaucoma medications, } \bar{x} \pm S D \\
\text { (range) }\end{array}$ & $1.8 \pm 1.4(0-4)$ \\
\hline $\begin{array}{l}\text { Baseline BCVA, LogMAR, } \bar{x} \pm S D \\
\text { (range) }\end{array}$ & $\mathrm{I} . \mathrm{I} \pm 0.75(0.3-3)$ \\
\hline Baseline IOP (mmHg), $\bar{x} \pm S D$ (range) & $15.9 \pm 3.6(11-25)$ \\
\hline $\begin{array}{l}\text { Baseline visual field } M D(d B), \bar{x} \pm S D \\
\text { (range)* }\end{array}$ & $-12.44 \pm 8.0(-30.57--2.20)$ \\
\hline Degrees of trabeculotomy & $325.3 \pm 59.5(100-360)$ \\
\hline
\end{tabular}

Note: *Visual field values were available from 22 eyes.

Abbreviations: $\bar{x}$, mean; SD, standard deviation; MD, mean deviation; $\mathrm{dB}$, decibels. 


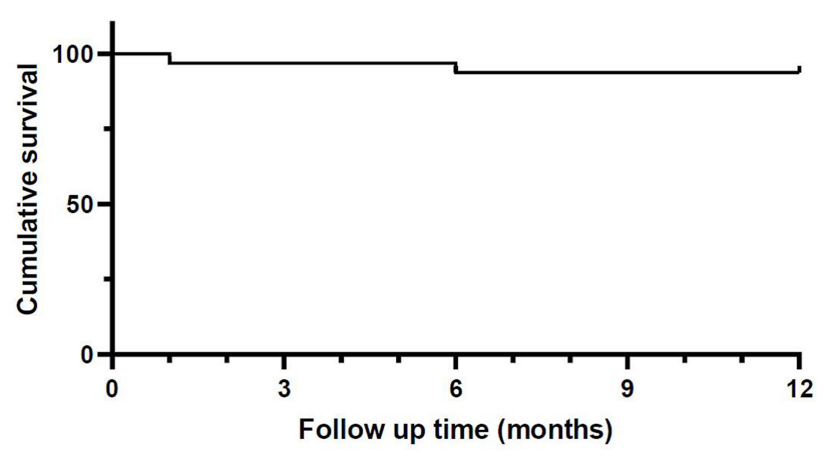

Figure I Kaplan-Meier survival curve demonstrating failure at different follow-up periods. Failure was defined as either IOP $>18 \mathrm{mmHg},<5 \mathrm{mmHg}$ or less than $20 \%$ reduction from baseline at any postoperative visit or need for additional surgery.

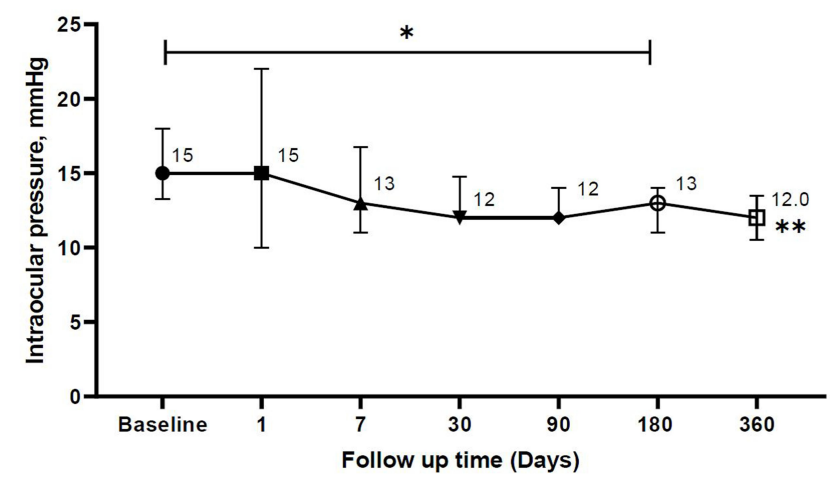

Figure 2 Figure showing the trend of intraocular pressure (median $\pm I Q R$ ) through time until 12 months of follow-up. *Wilcoxon signed-rank test $(p<0.0001)$. **Only $15 \%$ of eyes completed the follow-up time.

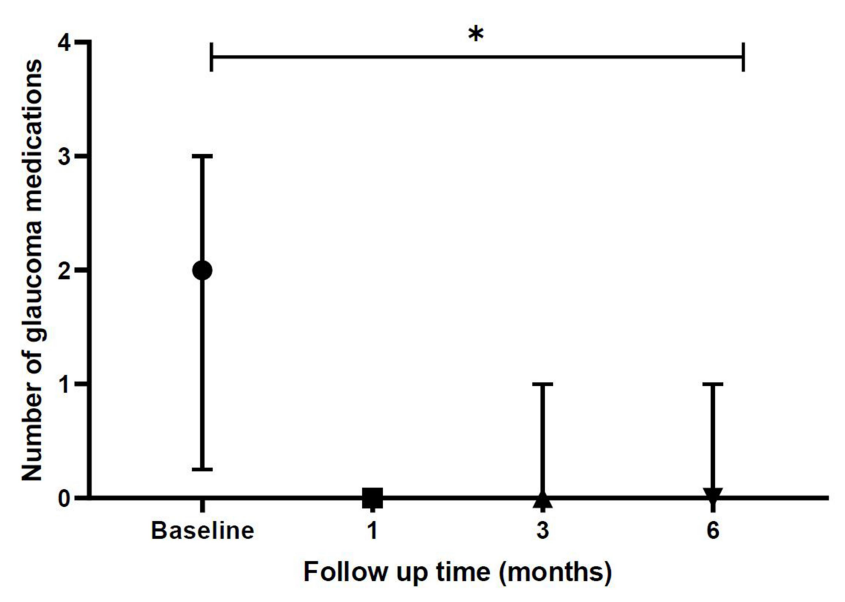

Figure 3 Change in the number of medications (median $\pm \mathrm{IQR}$ ) over time up to 6 months. *Wilcoxon signed-rank test $(\mathrm{p}<0.000 \mathrm{I})$.

$26.7 \%(\mathrm{n}=32$ eyes $)$ at the first month, $-18.1 \pm 16.2 \%(\mathrm{n}=$ 32 eyes) at 3 months, $-18.6 \pm 14.9 \%(n=32$ eyes $)$ at 6 months, and $-21.8 \pm 14.9 \%(\mathrm{n}=5$ eyes $)$ at 12 months after surgery. Table 2 summarizes various parameters evaluated through the follow-up. Figure 4 shows a scattergram of preoperative and postoperative 6-month IOP with sloped lines of $20 \%$ reduction from the IOP baseline. In relation to the BCVA, there was a clinically and statistically significant improvement (from $1.1 \pm 0.7$ to $0.4 \pm 0.7$ ) at 6 months $(\mathrm{p}<0.0001)$. At 12 months only 5 eyes $(15.7 \%)$ completed the follow-up. From 32 eyes, 30 (93.7\%) had a 270 to 360 -degree trabeculotomy and 2 (6.3\%) had a partial trabeculotomy (less than 270 degrees); the mean baseline visual field mean deviation (MD) was $-12.36 \pm 8.4$ $\mathrm{dB}$ and $-12.72 \pm 7.4 \mathrm{~dB}$ for the complete and partial trabeculotomy, respectively. IOP spikes and transient hyphema were the most common postoperative complications occurring in $18.7 \%$ and $9.3 \%$ of eyes, respectively, no patient required an anterior chamber washout.

\section{Discussion}

Conjunctival and scleral sparing techniques such as GATT have a short surgical time and effectively reduces IOP with minor complications related to the absence of a conjunctival bleb, making this procedure suitable for the treatment of many open-angle glaucoma types. ${ }^{12}$ Furthermore, this procedure may rescue the natural outflow system and allow future procedures, making it a suitable surgical option.

GATT is a minimal-invasive ab interno procedure with a reported mean IOP reduction from $33 \%$ to $65 \%$ at 12 months ${ }^{13-19}$ and a success rate (depending on various criteria) ranging from $63 \%$ to $89 \%$ at 12 and 24 months, respectively. ${ }^{13,15,17}$ Our results regarding treatment success $(65 \%)$ are consistent with the aforementioned reports. We found an IOP reduction of $18 \%$ and $21 \%$ at 6 and 12 months, respectively, which is lower than that reported in previous studies; this could be because baseline mean IOP values in the our study were not too high (15.9 \pm 3.6 $\mathrm{mmHg}$ ). Another possible explanation could be related to the fact that nearly a quarter $(22 \%)$ of patients had trabeculotomy of $270^{\circ}$ or less.

The cumulative proportion of failure in our study (6.3\% at 6 and 12 months) is consistent with several reports showing failure rates from 3.1 to $9 \%$. $^{13,18,19}$ Higher failure rates $(18-40 \%)$ are seen in eyes with previous glaucoma filtrating surgery or more advanced glaucoma. ${ }^{13,20}$ It has been reported that eyes with more advanced disease (visual field MD worse than $-15 \mathrm{~dB}$ ) ${ }^{18}$ had a higher risk of surgical failure, and some authors believe to be due to an atrophy of the collector channels. ${ }^{15}$ Lower failure rates were observed with combined cataract surgery and GATT, Grover et al reported 
Table 2 Evaluated Parameters at Consecutive Follow-Up Visits $(n=32)$

\begin{tabular}{|c|c|c|c|c|c|c|c|}
\hline Variable & Baseline & Day I & Day 7 & Month I & Month 3 & Month 6 & Month 12 \\
\hline Eyes, $\mathrm{n}$ & 32 & 23 & 32 & 32 & 32 & 32 & 5 \\
\hline Lost during follow-up, \% & 0 & 31.2 & 0 & 0 & 0 & 0 & 84.3 \\
\hline $\mathrm{IOP}(\mathrm{mm} \mathrm{Hg}), \bar{x} \pm \mathrm{SD}$ & $15.9 \pm 3.6$ & $17.4 \pm 10.4$ & $15.5 \pm 8.3$ & $13.2 \pm 3.3$ & $12.6 \pm 1.6$ & $12.5 \pm 1.4$ & $12.0 \pm 1.6$ \\
\hline IOP (mm Hg), median (IQR) & $15(13.2-18)$ & $15(10-22)$ & $13(11-16.7)$ & $12(12-15)$ & $12(12-14)$ & $13(11-14)$ & $12(10-13)$ \\
\hline IOP reduction,\% & NA & 8 & 3 & 13.3 & 18.1 & 18.6 & 21.8 \\
\hline Eyes with a $>20 \%$ IOP reduction, $\%$ & NA & 47.8 & 43.7 & 50 & 46.8 & 43.7 & 60 \\
\hline Percentage of complete success, $\%$ & NA & NA & NA & 56.2 & 68.7 & 65.6 & - \\
\hline Number of glaucoma medications, $\bar{x} \pm \mathrm{SD}$ & $1.8 \pm 1.4$ & NA & NA & $0.46 \pm 1$ & $0.65 \pm 1$ & $0.59 \pm 1$ & - \\
\hline $\begin{array}{l}\text { Number of glaucoma medications, median } \\
\text { (IQR) }\end{array}$ & $2(0.25-3)$ & & & $0(0-0)$ & $0(0-1)$ & $0(0-1)$ & \\
\hline
\end{tabular}

Abbreviations: $\bar{x}$, mean; SD, standard deviation; IOP, intraocular pressure; IQR, interquartile range; NA, no applicable.

that phakic eyes and those with prior cataract surgery required further glaucoma surgeries compared to patients who underwent a combined procedure. ${ }^{18}$ Cataract extraction also lowers IOP through several mechanisms. These include an increase in angle width, anatomic changes in the anterior chamber with traction on the ciliary body through the zonules and changes in the trabecular meshwork associated with induced inflammation and the subsequent increases on prostaglandin F2 $\alpha$ levels. ${ }^{21-24}$ It is not well established whether combined surgery with GATT has an additional effect on reducing the IOP.

Our combined surgery technique is different from that reported by Grover et al, ${ }^{18}$ because cataract extraction with phacoemulsification and intraocular lens implantation was performed first followed by GATT. The author prefers to perform GATT surgery after removing the cataract because it deepens the anterior chamber improving visualization of angle structures and surgical maneuverability. Additionally, the use of trypan blue dye in some patients may also stain the trabecular meshwork, facilitating its identification. A disadvantage of the procedure is that corneal edema may occur after cataract surgery, making the anterior segment difficult to see for GATT procedure. The mean baseline cup/disc ratio and visual field MD was $0.82 \pm 0.10(0.8-0.99)$ and $-12.44 \pm 8.0 \mathrm{~dB}(-30.57--2.20)$, respectively. Many patients were in an advanced stage of the disease and the reliability of MD values was low, additionally, visual field evaluation was only performed in $22(68.7 \%)$ eyes which is

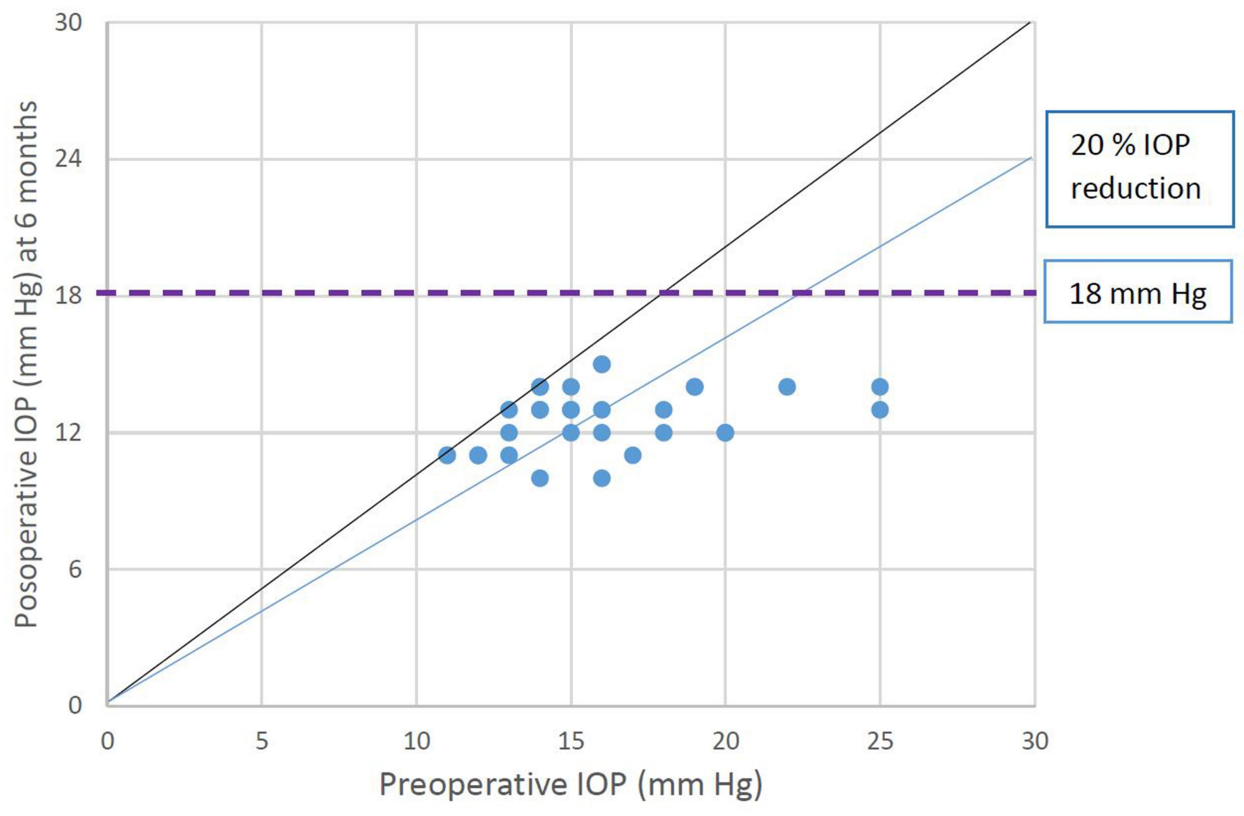

Figure 4 Scatter plot of preoperative and postoperative intraocular pressure (IOP) after 6 months. Each eye is illustrated as a circle. The oblique black line indicates no change of IOP. Circles above the black line define a higher postoperative IOP. Eyes below the line of $18 \mathrm{mmHg}$ or $20 \%$ IOP reduction fulfilled criteria of qualified success. 
one limitation of our study. Grover et al found that eyes with advanced disease (visual field MD worse than $-15 \mathrm{~dB}$ ) had a higher risk of failure at 2 years after GATT. ${ }^{18}$ It is hypothesized that the atrophy of the collector channel system may be the reason for failure since it was reported that in advanced glaucoma, the intra-scleral plexus and collector channels are destroyed and sclerotic. ${ }^{25}$ In our study, we could not evaluate an association between visual field MD values and surgical failure since many patients (31.3\%) did not have a baseline visual field assessment.

Some studies did not find any significant difference in the IOP reduction between trabeculotomy and combined GATT surgery. ${ }^{18,26}$ Since no comparison with eyes that underwent GATT alone was made, we cannot determine whether cataract surgery had any additional effect on the IOP reduction. No serious complications were reported, and as other studies showed, the most frequent complications observed were postoperative hyphema and IOP spikes. ${ }^{17,18,20}$ The overall safety profile of the procedure was good, previously reported hyphema rate when OVD was left at the end of the surgery ranged from $28 \%$ to $38 \%{ }^{13,15,16,18}$ and when no OVD was left was $70 \%,{ }^{27}$ which is markedly higher compared to our report $(9.3 \%)$; this could be due to the fact that there was variability on how hyphema was documented. Cases with microhyphema (small hyphema characterized by suspended red blood cells in the anterior chamber without the formation of a layered clot) were not recorded in our study, it is possible that our overall rate of hyphema was higher because the anterior chamber was not filled with viscoelastic at the end of the surgery. IOP spikes reported may vary among studies and techniques, in eyes in which a $25 \%$ anterior chamber was filled with OVD at the end of the surgery, the rate of IOP peaks vary from $2-24.7 \%{ }^{11,13,15}$ and in the study in which no OVD was left, it was $5.4 \%,{ }^{27}$ which is lower from our results $(18.7 \%)$. It is possible that our rate of IOP peaks was higher, since at the end of the surgery the anterior chamber was completely filled with BSS leaving the eye hypertensive, additionally most of the eyes had an advanced stage of the disease (mean visual field MD value of $-12.44 \pm 8.0 \mathrm{~dB}$ ) which could have affected the outflow of BSS. Similar to Cubuk et al, we did not leave any OVD at the end of the surgery because we agree with the authors and believe that filling the anterior chamber (to any degree) with an OVD could produce IOP spikes that could cause detrimental effects on the optic nerve fiber layer especially in patients with advanced glaucoma, so we decided not to fill the anterior chamber with an OVD.
The extent (clock hours) and the degree of blanching of the EVFW were not evaluated since they were not reported in clinical charts. A limited EVFW has been correlated with surgical failure and a higher IOP level after surgery. ${ }^{28}$ Fellman et al described the EVFW as a perioperative marker for the success of the Trabectome surgery and they found that eyes with a limited or absent EVFW were more likely to have higher postoperative IOP levels and to require more glaucoma medications. ${ }^{29}$ This negative correlation between postoperative IOP and the extent of the EVFW was also found by Aktas et al suggesting that the extension of EVFW could be a prognostic indicator for the surgical success of GATT surgery. ${ }^{28}$ However, the author believes that the observation of blood reflux with accumulation of blood in Schlemm's canal after cataract surgery and just before the GATT procedure may be an indicator that the collector channels are functional.

Limitations such as the small sample size, its retrospective nature, the lack of a control group, and the limited follow-up time weaken our report. However, our study may contribute to the body of evidence reporting the IOP lowering effect and safety of GATT as a viable option for combined procedures. Additionally, we report a modification from the original technique that could be explored in future investigations. A control group in the setting of a randomized clinical trial is needed to assess the advantages of this procedure; however, it is out of the scope of our study, and it will be considered in future research.

\section{Conclusion}

Combined GATT with cataract surgery consistently reduced the IOP and was associated with low failure surgical rates and few ocular complications. This procedure offers the advantage to avoid the need for a bleb, scleral, or conjunctival incision, allowing the possibility for future glaucoma procedures. Clinical trials are needed to better understand its real long-term efficacy and safety.

\section{Ethics}

Institution Responsible for the Approval of the Research Ethics Committee:

RCEI-56: Ethics committee from the National Eye Institute (INO), Lima, Peru.

\section{Acknowledgments}

We gratefully thank the patients for participation in this study.

\section{Author Contributions}

All authors made a significant contribution to the work reported, whether that is in the conception, study design, 
execution, acquisition of data, analysis and interpretation, or in all these areas; took part in drafting, revising or critically reviewing the article; gave final approval of the version to be published; have agreed on the journal to which the article has been submitted; and agree to be accountable for all aspects of the work.hip.

\section{Funding}

The authors received no financial support for the research, authorship, or publication of this article. .

\section{Disclosure}

The author declared no potential conflicts of interest with respect to the research, authorship, or publication of this article.

\section{References}

1. Quigley HA, Broman AT. The number of people with glaucoma worldwide in 2010 and 2020. Br J Ophthalmol. 2006;90 (3):262-267. doi:10.1136/bjo.2005.081224

2. Leske MC, Heijl A, Hyman L, Bengtsson B. Early manifest glaucoma trial: design and baseline data. Ophthalmology. 1999;106 (11):2144-2153. doi:10.1016/S0161-6420(99)90497-9

3. Kass MA, Heuer DK, Higginbotham EJ, et al. The ocular hypertension treatment study: a randomized trial determines that topical ocular hypotensive medication delays or prevents the onset of primary open-angle glaucoma. Arch Ophthalmol. 2002;120(6):701-713. doi:10.1001/archopht.120.6.701

4. Glaucoma Laser Trial Research Group. The glaucoma laser trial (GLT) and glaucoma laser trial follow-up study: 7. Results. Am J Ophthalmol. 1995;120(6):718-731. doi:10.1016/S0002-9394(14)72725-4

5. Lichter PR, Musch DC, Gillespie BW, et al. Interim clinical outcomes in the collaborative initial glaucoma treatment study comparing initial treatment randomized to medications or surgery. Ophthalmology. 2001;108(11):1943-1953. doi:10.1016/S0161-6420 (01)00873-9

6. Smith R. A new technique for opening the canal of Schlemm. Preliminary report. $\mathrm{Br} J$ Ophthalmol. 1960;44:370-373. doi:10.11 36/bjo.44.6.370

7. Harms H, Dannheim R. Epicritical consideration of 300 cases of trabeculotomy "ab externo.". Trans Ophthalmol Soc UK. 1970;89: 491-499.

8. Allen L, Burian HM. Trabeculotomy ab externo. A new glaucoma operation: technique and results of experimental surgery. $\mathrm{Am}$ J Ophthalmol. 1962;53:19-26. doi:10.1016/0002-9394(62)90388-4

9. McPherson SD, McFarland D. External trabeculotomy for developmental glaucoma. Ophthalmology. 1980;87(4):302-305.

10. Bonnet M, Schiffer HP. [Trabeculotomy ab externo: localization of Schlemm's canal through catheterization of an aqueous-humor vein] Klin Monbl Augenheilkd. 1972;161(5):563-566.[Article in German].

11. Grover DS, Godfrey DG, Smith O, Feuer WJ, Montes de Oca I, Fellman RL. Gonioscopy-assisted transluminal trabeculotomy, ab interno trabeculotomy: technique report and preliminary results. Ophthalmology. 2014;121(4):855-861. doi:10.1016/j.ophtha.2013.11.001

12. Grover DS, Smith O, Fellman RL, et al. Gonioscopy assisted transluminal trabeculotomy: an ab interno circumferential trabeculotomy for the treatment of primary congenital glaucoma and juvenile open angle glaucoma. Br J Ophthalmol. 2015;99(8):1092-1096. doi:10.11 36/bjophthalmol-2014-306269
13. Rahmatnejad K, Pruzan NL, Amanullah S, et al. Surgical outcomes of gonioscopy-assisted transluminal trabeculotomy (GATT) in patients with open-angle glaucoma. J Glaucoma. 2017;26(12):11 37-1143. doi:10.1097/IJG.0000000000000802

14. Quan AV, Yannuzzi NA, Chen J, Wang YE, Townsend JH, Chang TC. Gonioscopy-assisted transluminal trabeculotomy (GATT) in patients with secondary open-angle glaucoma following vitreoretinal surgery. J Glaucoma. 2020;29(4):e23-5. doi:10.1097/ IJG.0000000000001461

15. Aktas Z, Ucgul AY, Bektas C, Sahin Karamert S. Surgical outcomes of prolene gonioscopy-assisted transluminal trabeculotomy in patients with moderate to advanced open-angle glaucoma. J Glaucoma. 2019;28 (10):884-888. doi:10.1097/IJG.0000000000001331

16. Boese EA, Shah M. Gonioscopy-assisted transluminal trabeculotomy (GATT) is an effective procedure for steroid-induced glaucoma. J Glaucoma. 2019;28(9):803-807. doi:10.1097/IJG.000000000000 1317

17. Sato T, Kawaji T, Hirata A, Mizoguchi T. 360-degree suture trabeculotomy ab interno with phacoemulsification in open-angle glaucoma and coexisting cataract: a pilot study. BMJ Open Ophthalmol. 2018;3(1):e000159. doi:10.1136/bmjophth-2018-000159

18. Grover DS, Smith O, Fellman RL, et al. Gonioscopy-assisted transluminal trabeculotomy: an $\mathrm{Ab}$ interno circumferential trabeculotomy: 24 months follow-up. J Glaucoma. 2018;27(5):393-401. doi:10.10 97/IJG.0000000000000956

19. Baykara M, Poroy C, Erseven C. Surgical outcomes of combined gonioscopy-assisted transluminal trabeculotomy and cataract surgery. Indian J Ophthalmol.2019;67(4):505-508. doi:10.4103/ijo.IJO_1007_18

20. Grover DS, Godfrey DG, Smith O, Shi W, Feuer WJ, Fellman RL. Outcomes of gonioscopy-assisted transluminal trabeculotomy (GATT) in eyes with prior incisional glaucoma surgery. J Glaucoma. 2017;26(1):41-45. doi:10.1097/IJG.0000000000000564

21. Kung JS, Choi DY, Cheema AS, Singh K. Cataract surgery in the glaucoma patient. Middle East Afr J Ophthalmol. 2015;22(1):10-17. doi:10.4103/0974-9233.148343

22. Kerstetter JR, Brubaker RF, Wilson SE, Kullerstrand LJ. Prostaglandin F2 alpha-1-isopropylester lowers intraocular pressure without decreasing aqueous humor flow. Am J Ophthalmol. 1988;105 (1):30-34. doi:10.1016/0002-9394(88)90117-1

23. Shingleton BJ, Pasternack JJ, Hung JW, O'Donoghue MW. Three and five year changes in intraocular pressures after clear corneal phacoemulsification in open angle glaucoma patients, glaucoma suspects, and normal patients. J Glaucoma. 2006;15(6):494-498. doi:10.1097/ 01.ijg.0000212294.31411.92

24. Hayashi K, Hayashi H, Nakao F, Hayashi F. Changes in anterior chamber angle width and depth after intraocular lens implantation in eyes with glaucoma. Ophthalmology. 2000;107(4):698-703. doi:10.1016/S0161-6420(00)00007-5

25. Dvorak-Theobald G. Schlemm's canal: its anastomoses and anatomic relations. Trans Am Ophthalmol Soc. 1934;32:574-595.

26. Shinmei Y, Kijima R, Nitta T, et al. Modified 360-degree suture trabeculotomy combined with phacoemulsification and intraocular lens implantation for glaucoma and coexisting cataract. $J$ Cataract Refract Surg. 2016;42(11):1634-1641. doi:10.1016/j.jcrs.2016.08.016

27. Cubuk MO, Unsal E. One-year results of gonioscopy-assisted transluminal trabeculotomy: evaluation of prognostic factors. Eur J Ophthalmol. 2020;1120672120908716.

28. Aktas Z, Ozmen MC, Atalay HT, Ucgul AY. Evaluation of episcleral venous fluid wave during gonioscopy assisted transluminal trabeculotomy in patients with advanced glaucoma. Eye (Lond). 2019;33 (4):668-673. doi:10.1038/s41433-018-0254-5

29. Fellman RL, Feuer WJ, Grover DS. Episcleral venous fluid wave correlates with trabectome outcomes: intraoperative evaluation of the trabecular outflow pathway. Ophthalmology. 2015;122(12):23852391.e1. doi:10.1016/j.ophtha.2015.08.038 


\section{Publish your work in this journal}

Clinical Ophthalmology is an international, peer-reviewed journal covering all subspecialties within ophthalmology. Key topics include: Optometry; Visual science; Pharmacology and drug therapy in eye diseases; Basic Sciences; Primary and Secondary eye care; Patient Safety and Quality of Care Improvements. This journal is indexed on PubMed
Central and CAS, and is the official journal of The Society of Clinical Ophthalmology (SCO). The manuscript management system is completely online and includes a very quick and fair peer-review system, which is all easy to use. Visit http://www.dovepress.com/ testimonials.php to read real quotes from published authors. 